\title{
BIFURCATION MODEL OF SUCCESSIONS IN ECOSYSTEMS
}

\author{
Serge V. Chernyshenko \\ Roman V. Ruzich \\ Department of Applied Mathematics and Social Informatics \\ Khmelnitsky National University \\ 29000, Khmelnitsky, Ukraine \\ Email: svc@a-teleport.com; ninasus@gmail.com
}

\section{KEYWORDS}

Succession, biogeocoenose, system of ordinary differential equations, bifurcation.

\begin{abstract}
Models of the long-term ecological successions are considered. Succession process is considered as step-bystep changing of dominant association. The model of open Eigen's hypercycle has been used for modeling of the process. Qualitative analysis for three-dimension case has been carried out, and local bifurcations have been investigated. The process of succession can be interpreted as system's choosing a proper level of complexity (or dimension) depending on the capacity of environment (the size of ecological niche). Connections between changing a state of the system and bifurcations in phase space is shown.
\end{abstract}

\section{INTRODUCTION}

Processes, which take place in ecosystems, are extremely complex; their theoretical investigation should be based on abstract concepts, which describe some general properties of the systems on the global level. One of such theoretical simplifications is the concept of succession. The succession is considered as consecutive change of one ecosystem (phitocoenose, biogeocoenoe, etc.) by other in a certain area of environment. It is not a simple transformation, but a process of simplification of the structure of the ecosystem. Any state of a system can be characterised by dominate association of species (usually phototrophic), which are "amalgamated" by other satellite species by trophic relations. Such associations appear as the key elements of biogeocoenose.

Stochastic models (Culver 1981; Lippe et al. 1985; Logofet 1997; Lourival 2011) are often used for description of succession processes. The transition probability is basic parameter which determinate the dynamics of the system in such models. Such approach can be useful for simulation the system dynamics of the system, but does not reflect moving forces of the process. Concerning long-term successions, many researches (Sukachev 1972; Kogan 1977; Tilman 1990; Rabotnov 1992) emphasized essential role of competition for this processes. Use of models of competition of the Volterra type (Lepš and Prach 1981; Chakrabarti et al. 1995;
Chernyshenko 1995; Weis et al. 2007) for description of succession process looks as very reasonable.

Additionally to competition, there is evident positive influence of previous stages for next ones. Similar relationships between elements are described by the well-known model of hypercycle (Eigen and Schuster 1979). In the same time, relations between associations during succession have not cyclic character; the hypercycle should be open (Chernyshenko 2005). The model of open hypercycle is similar, but not equivalent to Lotka-Volterra models of the competition or "predator-prey" type. The model reflects a connection between final stage of succession (and corresponding level of complexity of the system) and the size of ecological niche. In the contribution a three-dimension case of the open hypercycle model is considered; change of the ecosystem state is interpreted mathematically as a consequence of bifurcations.

\section{OPEN EIGEN'S HYPERCYCLE}

Let's consider dynamics of ecosystem, which is described by the three-dimension open hypercycle model:

$$
\left\{\begin{array}{l}
\frac{d x_{1}}{d t}=\left(F_{1}(x)-\frac{1}{S_{0}}\left(x_{1} F_{1}(x)+x_{2} F_{2}(x)+x_{3} F_{3}(x)\right)\right) x_{1}, \\
\frac{d x_{2}}{d t}=\left(F_{2}(x)-\frac{1}{S_{0}}\left(x_{1} F_{1}(x)+x_{2} F_{2}(x)+x_{3} F_{3}(x)\right)\right) x_{2},(1) \\
\frac{d x_{3}}{d t}=\left(F_{3}(x)-\frac{1}{S_{0}}\left(x_{1} F_{1}(x)+x_{2} F_{2}(x)+x_{3} F_{3}(x)\right)\right) x_{3},
\end{array}\right.
$$

here $F_{1}(x)=N-x_{1}, F_{2}(x)=a_{1} x_{1}-x_{2}, F_{3}(x)=a_{2} x_{2}-x_{3}$ are known as Allen's functions (Allen 1976); $a_{1}>0$, $a_{2}>0, N>0, S_{0}>0, x_{1}(t), x_{2}(t), x_{3}(t)$ are sizes (biomasses) of the associations, $S_{0}$ is capacity of environment, $N$ is a coefficient which determine equilibrium size of the first association, when it develops alone; $a_{1}$ is a coefficient which describes a level of dependence of the second association from the first one, $a_{2}$ is the same for the third and second associations.

\section{THE EQUILIBRIUM POINTS OF THE SYSTEM (1)}

The system equilibrium points can be found as roots of the system of algebraic equations of the third order: 


$$
\left\{\begin{array}{l}
\left(F_{1}(x)-\frac{1}{S_{0}}\left(x_{1} F_{1}(x)+x_{2} F_{2}(x)+x_{3} F_{3}(x)\right)\right) x_{1}=0, \\
\left(F_{2}(x)-\frac{1}{S_{0}}\left(x_{1} F_{1}(x)+x_{2} F_{2}(x)+x_{3} F_{3}(x)\right)\right) x_{2}=0, \\
\left(F_{3}(x)-\frac{1}{S_{0}}\left(x_{1} F_{1}(x)+x_{2} F_{2}(x)+x_{3} F_{3}(x)\right)\right) x_{3}=0 .
\end{array}\right.
$$

The solution is trivial; there are 11 equilibrium points:

$$
\begin{aligned}
& P_{1}:(0,0,0) ; \quad P_{2}:(N, 0,0) ; \quad P_{3}:\left(N, a_{1} N, 0\right) ; \\
& P_{4}:\left(N, a_{1} N, a_{1} a_{2} N\right) ; \quad P_{5}:\left(S_{0}, 0,0\right) ; \quad P_{6}:\left(0, S_{0}, 0\right) ; \\
& P_{7}:\left(0,0, S_{0}\right) ; \quad P_{8}:\left(\frac{N+S_{0}}{a_{1}+2}, \frac{S_{0}\left(a_{1}+1\right)-N}{a_{1}+2}, 0\right) ; \\
& P_{9}:\left(\frac{S_{0}+N}{2}, 0, \frac{S_{0}-N}{2}\right) ; P_{10}:\left(0, \frac{S_{0}}{a_{2}+2}, \frac{S_{0}\left(a_{2}+1\right)}{a_{2}+2}\right) ; \\
& P_{11}:\left(\frac{S_{0}+N\left(a_{2}+2\right)}{a_{1} a_{2}+a_{1}+a_{2}+3}, \frac{\left(a_{1}+1\right) S_{0}+N\left(a_{1}-1\right)}{a_{1} a_{2}+a_{1}+a_{2}+3},\right. \\
&\left.\frac{\left(a_{1} a_{2}+a_{2}+1\right) S_{0}-N\left(a_{1}+a_{2}+1\right)}{a_{1} a_{2}+a_{1}+a_{2}+3}\right) .
\end{aligned}
$$

One of the signs of system bifurcations is merging of equilibrium points. It is easily possible to find conditions for merging of couples of the points:

$$
\begin{aligned}
& P_{5} \text { and } P_{8} \text {, if } \frac{S_{0}}{N}=\frac{1}{a_{1}+1} ; \\
& P_{8} \text { and } P_{11}, \text { if } \frac{S_{0}}{N}=\frac{a_{1}+a_{2}+1}{a_{1} a_{2}+a_{2}+1} ; \\
& P_{9} \text { and } P_{11} \text {, if }\left\{\begin{array}{l}
\frac{S_{0}}{N}=\frac{1-a_{1}}{a_{1}+1} \\
a_{1} \in(0,1) ;
\end{array}\right. \\
& P_{2}, P_{5} \text { and } P_{9}, \text { if } \frac{S_{0}}{N}=1 ; \\
& P_{3} \text { and } P_{8}, \text { if } \frac{S_{0}}{N}=a_{1}+1 ; \\
& P_{4} \text { and } P_{11} \text {, if } \frac{S_{0}}{N}=a_{1} a_{2}+a_{1}+1 .
\end{aligned}
$$

Hypothesis 1. In the system (1) the bifurcation values of parameters are following:

$$
\begin{array}{lll}
\frac{S_{0}}{N}=\frac{1-a_{1}}{a_{1}+1}, \text { when } a_{1} \in(0,1) ; & \frac{S_{0}}{N}=\frac{1}{a_{1}+1} ; \\
\frac{S_{0}}{N}=\frac{a_{1}+a_{2}+1}{a_{1} a_{2}+a_{2}+1} ; \quad \frac{S_{0}}{N}=1 ; & \frac{S_{0}}{N}=a_{1}+1 ; \\
\frac{S_{0}}{N}=a_{1} a_{2}+a_{1}+1 . & &
\end{array}
$$

\section{ANALYSIS OF THE EQUILIBRIUM POINTS}

Let's consider the Jacobi matrix of the system (1):

$$
\begin{aligned}
& J=\left[\begin{array}{lll}
m_{11} & m_{12} & m_{13} \\
m_{21} & m_{22} & m_{23} \\
m_{31} & m_{32} & m_{33}
\end{array}\right], \\
& m_{11}=\frac{3}{S_{0}} x_{1}^{2}-2 \frac{N+S_{0}+a_{1} x_{2}}{S_{0}} x_{1}+\frac{N S_{0}+x_{2}^{2}}{S_{0}}+\frac{x_{3}^{2}-a_{2} x_{3} x_{2}}{S_{0}} \\
& m_{12}=\frac{2 x_{2}-a_{2} x_{3}}{S_{0}} x_{1}-\frac{a_{1}}{S_{0}} x_{1}^{2}, m_{13}=\frac{2 x_{3}-a_{2} x_{2}}{S_{0}} x_{1}, \\
& m_{21}= \frac{a_{1} S_{0}-N+2 x_{1}}{S_{0}} x_{2}-\frac{a_{1}}{S_{0}} x_{2}^{2}, \\
& m_{22}= \frac{a_{1} S_{0}-N-2 a_{1} x_{2}}{S_{0}} x_{1}+\frac{1}{S_{0}} x_{1}^{2}-\frac{2 S_{0}+2 a_{2} x_{3}}{S_{0}} x_{2}+\frac{x_{3}^{2}+3 x_{2}^{2}}{S_{0}} \\
& m_{23}= \frac{2 x_{3}-a_{2} x_{2}}{S_{0}} x_{2}, m_{31}=\frac{2 x_{1}-a_{1} x_{2}-N}{S_{0}} x_{3}, \\
& m_{32}= \frac{2 x_{2}+a_{2} S_{0}-a_{1} x_{1}}{S_{0}} x_{3}-\frac{a_{2}}{S_{0}} x_{3}^{2}, \\
& m_{33}=-\frac{N+a_{1} x_{2}}{S_{0}} x_{1}+\frac{a_{2} S_{0}-2 a_{2} x_{3}}{S_{0}} x_{2}+ \\
&+\frac{1}{S_{0}} x_{1}^{2}+\frac{1}{S_{0}} x_{2}^{2}-2 x_{3}+\frac{3}{S_{0}} x_{3}^{2} .
\end{aligned}
$$

Eigenvalues of the matrix (2) in the stationary points: for $P_{1}$ they are $\lambda_{1}=N, \lambda_{2}=0, \lambda_{3}=0$;

for $P_{2}$ they are $\lambda_{1}=\frac{N^{2}}{S_{0}}-N, \lambda_{2}=N a_{1}, \lambda_{3}=0$; for $P_{3}$ they are $\lambda_{1}=a_{1} a_{2} N \quad \lambda_{2,3}=\frac{N}{2 S_{0}}\left(N-S_{0}\left(a_{1}+1\right) \pm\right.$

$$
\left.\pm \sqrt{N^{2}+2 N S_{0}\left(2 a_{1}^{2}+a_{1}-1\right)+S_{0}^{2}\left(a_{1}-1\right)^{2}}\right)
$$

for $P_{4}$ an analytical form of the eigenvalues was not found. (The Descartes method (Berezin and Zitkov 1959) is used for determination of the stress intervals of the eigenvalues. Three eigenvalues are negative, when $S_{0} / N \in\left(1+a_{1}+a_{1} a_{2} ;+\infty\right)$; two negative and one positive when $\left.S_{0} / N \in\left(0 ; 1+a_{1}+a_{1} a_{2}\right)\right)$;

for $P_{5}$ they are $\lambda_{1}, \lambda_{3}=S_{0}-N, \lambda_{2}=S_{0}\left(1+a_{1}\right)-N$; for $P_{6}$ they are $\lambda_{1}=N+S_{0}, \lambda_{2}=S_{0}\left(a_{2}+1\right), \lambda_{3}=S_{0}$; for $P_{7}$ they are $\lambda_{1}=N+S_{0}, \lambda_{2,3}=S_{0}$; for $P_{8}$ they are

$$
\begin{aligned}
& \lambda_{1}=-\frac{N\left(a_{1}+1\right)-S_{0}}{a_{1}+2}, \lambda_{2}=-\frac{\left(N+S_{0}\right)\left(S_{0}\left(a_{1}+1\right)-N\right)}{S_{0}\left(a_{1}+2\right)}, \\
& \lambda_{3}=-\frac{N\left(1+a_{1}+a_{2}\right)-S_{0}\left(1+a_{2}+a_{1} a_{2}\right)}{a_{1}+2} ;
\end{aligned}
$$

for $P_{9}$ they are $\lambda_{1}=\frac{N^{2}-S_{0}^{2}}{2 S_{0}}, \lambda_{2}=\frac{S_{0}-N}{2}$,

$$
\lambda_{3}=\frac{S_{0}\left(a_{1}+1\right)+N\left(a_{1}-1\right)}{2} ;
$$


for $P_{10}$ they are $\lambda_{1}=-\frac{S_{0}\left(a_{2}+1\right)}{a_{2}+2}, \lambda_{2}=\frac{S_{0}}{S_{2}+2}$,

$$
\lambda_{3}=\frac{S_{0}+N\left(a_{2}+2\right)}{a_{2}+2} ;
$$

for $P_{11}$ one eigenvalue is $\lambda_{1}=\frac{S_{0}-N\left(1+a_{1}+a_{1} a_{2}\right)}{a_{1}+a_{2}+a_{1} a_{2}+3}$, the others are roots of a quadratic equation. The analysis of the equation shown that there is a pair of eigenvalues of different sign, if

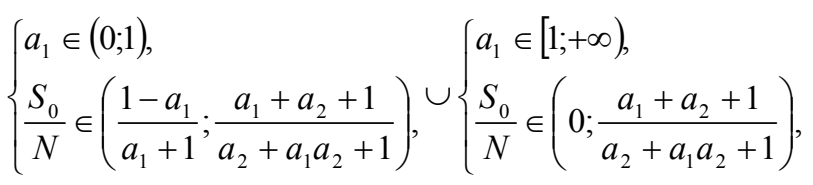

a pair of positive eigenvalues, if

$$
\left\{\begin{array}{l}
a_{1} \in(0 ; 1), \\
\frac{S_{0}}{N} \in\left(0 ; \frac{1-a_{1}}{a_{1}+1}\right)
\end{array}\right.
$$

a pair of negative eigenvalues, if

$$
\frac{S_{0}}{N} \in\left(\frac{a_{1}+a_{2}+1}{a_{2}+a_{1} a_{2}+1} ;+\infty\right) \text {. }
$$

On the base of these results one can prove that:

$P_{1}$ and $P_{2}$ are unstable complicated equilibrium points;

$P_{3}$ is a saddle with two-dimension unstable subspace, if $S_{0} / N \in\left(0, a_{1}+1\right)$, or two-dimension stable subspace, if $S_{0} / N \in\left(a_{1}+1,+\infty\right)$;

$P_{4}$ is a saddle with two-dimension stable subspace, if $S_{0} / N \in\left(0,1+a_{1}+a_{1} a_{2}\right)$, or stable equilibrium point (node or spiral point) if $S_{0} / N \in\left(1+a_{1}+a_{1} a_{2},+\infty\right)$;

$P_{5}$ is a stable node, if $S_{0} / N \in\left(0,\left(a_{1}+1\right)^{-1}\right)$, a saddle with two-dimension stable subspace, if $S_{0} / N \in\left(\left(a_{1}+1\right)^{-1}, 1\right)$, and an unstable node, if $S_{0} / N \in(1,+\infty)$;

$P_{6}$ and $P_{7}$ are unstable nodes;

$P_{8}$ is a saddle with two-dimension stable subspace, if $\frac{S_{0}}{N} \in\left(0, \frac{1}{a_{1}+1}\right) \cup\left(\frac{1+a_{1}+a_{2}}{1+a_{2}+a_{1} a_{2}}, 1+a_{1}\right)$, a stable node, if $\frac{S_{0}}{N} \in\left(\frac{1}{a_{1}+1}, \frac{1+a_{1}+a_{2}}{1+a_{2}+a_{1} a_{2}}\right)$, and a saddle with twodimension unstable subspace, if $S_{0} / N \in\left(1+a_{1},+\infty\right)$; $P_{9}$ is a saddle with two-dimension unstable subspace, if

$$
\left\{\begin{array}{l}
\frac{S_{0}}{N} \in\left(\frac{1-a_{1}}{1+a_{1}}, 1\right) \cup(1,+\infty), \\
a_{1} \in(0,1),
\end{array}\right.
$$

and with two-dimension stable subspace, if

$$
S_{0} / N \in\left(0,\left(1-a_{1}\right) /\left(1+a_{1}\right)\right), \quad a_{1} \in(0,1),
$$

$P_{10}$ is a saddle with two-dimension unstable subspace; $P_{11}$ is a saddle with two-dimension unstable subspace, if

$$
S_{0} / N \in\left(0,\left(1-a_{1}\right) /\left(1+a_{1}\right)\right), \quad a_{1} \in(0,1),
$$

or a saddle with two-dimension unstable subspace, if

$$
\begin{aligned}
& \left\{\frac{S_{0}}{N} \in\left(\frac{1-a_{1}}{a_{2}+1}, \frac{a_{1}+a_{2}+1}{a_{2}+a_{1} a_{2}+1}\right) ; a_{1} \in(0,1)\right\} \cup \\
& \cup\left\{\begin{array}{l}
a_{1} \in[1,+\infty), \\
\frac{S_{0}}{N} \in\left(0, \frac{a_{1}+a_{2}+1}{a_{2}+a_{1} a_{2}+1}\right), \cup \frac{S_{0}}{N} \in\left(a_{1}+a_{1} a_{2}+1,+\infty\right)
\end{array}\right.
\end{aligned}
$$

or a stable equilibrium point (node or spiral point) if

$$
\frac{S_{0}}{N} \in\left(\frac{a_{1}+a_{2}+1}{1+a_{2}+a_{1} a_{2}}, 1+a_{1}+a_{1} a_{2}\right) .
$$

\section{BIFURCATION POINTS}

Let's determinate bifurcation values of parameters on the base of analysis of equilibrium points. The bifurcation diagram is presented in Fig.1.

For values of the parameters from the regions I, II, III, IV, there are two complicated equilibrium points, two unstable nodes, three saddles with two-dimension unstable subspace, three saddles with two-dimension stable subspace and one stable node.

For values of the parameters from the regions V, VI, VII, VIII, there are two complicated equilibrium points, three unstable node, three saddles with two-dimension unstable subspace, two saddles with two-dimension stable subspace and one stable node in phase space.

When quotient of the parameters $S_{0}$ and $N$ pass through value 1 , one saddle disappears and unstable node appears. Let's consider the phase portrait in the first octant, because of its importance for practical applications. Let's note that there are not equilibrium points, which belongs the first octant for any values of the parameters. Thus, the point $P_{8}$ belongs to the first octant, when $\frac{S_{0}}{N} \geq \frac{1}{1+a_{1}}$, point $P_{9}-$ when $\frac{S_{0}}{N} \geq 1$, point $P_{11}-$ when $\frac{S_{0}}{N} \geq \frac{1+a_{1}+a_{2}}{1+a_{2}+a_{1} a_{2}}$. One can see that when $\frac{S_{0}}{N}$ pass through these tree values, topology of the first 
octant change. It is possible to summarise the obtained results by a theorem.

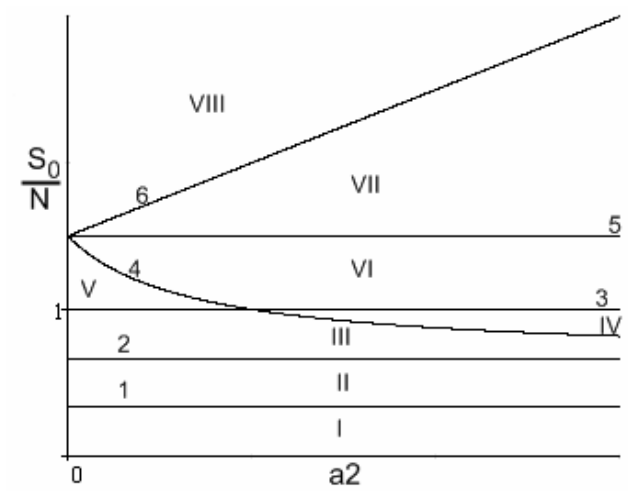

a) $a_{1} \in(0,1)$

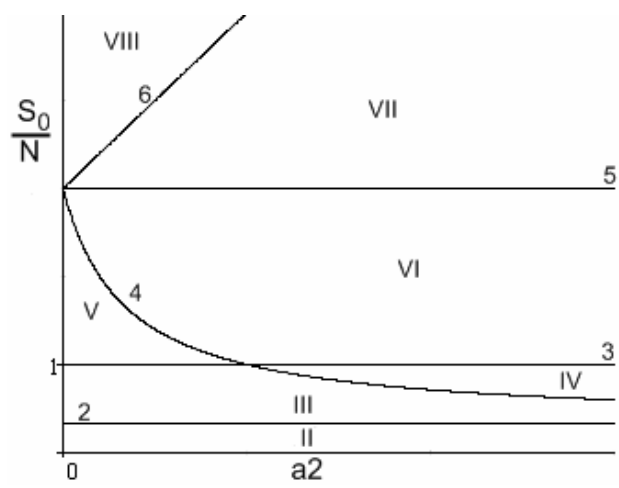

б) $a_{1} \in[1,+\infty)$

Figure 1: Sections of the parametric surfaces

(1. $\frac{S_{0}}{N}=\frac{1-a_{1}}{a_{1}+1}, \quad 2 \cdot \frac{S_{0}}{N}=\frac{1}{1+a_{1}}, \quad 3 \cdot \frac{S_{0}}{N}=1$,

4. $\left.\frac{S_{0}}{N}=\frac{1+a_{1}+a_{2}}{1+a_{2}+a_{1} a_{2}}, 5 \cdot \frac{S_{0}}{N}=a_{1}+1,6 \cdot \frac{S_{0}}{N}=1+a_{1}+a_{1} a_{2}\right)$

Theorem 1. There are following bifurcation values of parameters for the system (1):

1) $S_{0} / N=1$ (for the all phase space);

2) $\frac{S_{0}}{N}=\frac{1}{1+a_{1}}, \frac{S_{0}}{N}=1, \frac{S_{0}}{N}=\frac{1+a_{1}+a_{2}}{1+a_{2}+a_{1} a_{2}}$ (for the first octant).

Now it is clear, that the Hypothesis 1 is partly wrong. Thus, the equation $\frac{S_{0}}{N}=\frac{1-a_{1}}{a_{1}+1}\left(\right.$ when $\left.a_{1} \in(0,1)\right)$ does not correspond to a bifurcation. When the quotient of the parameters exceeds this value, two-dimension stable subspace of the point $P_{9}$ becomes one-dimension and, contra versa, one-dimension stable subspace of the point $P_{11}$ becomes two-dimension.

The similar situation takes place for the equation $S_{0} / N=1+a_{1}+a_{1} a_{2}$ : the point $P_{4}$ becomes a stable node (spiral point), whereas the point $P_{11}$ changes type from a stable node (spiral point) to a saddle. The topology of the phase space does not change; therefore, there is not a bifurcation for these values of parameters. A special situation is connected with the equation $S_{0} / N=1$. In this case three equilibrium points $\left(P_{2}\right.$, $\left.P_{5}, P_{9}\right)$ merge into one. When parameters pass through this point, stability (and type) of the equilibrium point $P_{9}$ does not change, whereas the point $P_{5}$ becomes unstable node and two-dimension unstable subspace of the point $P_{2}$ becomes one-dimension. It can be shown that the bifurcation takes place in the first octant.

Let's consider the set of equilibrium points which have two nonzero components: the first and the $j$-th $(j>2)$.

One can find coordinates of such equilibrium points from the system of algebraic equations:

$$
\left\{\begin{array}{l}
\left(N-x_{1}-\left(\left(N-x_{1}\right) x_{1}-x_{j}^{2}\right) / S_{0}\right) x_{1}=0, \\
\left(-x_{j}-\left(\left(N-x_{1}\right) x_{1}-x_{j}^{2}\right) / S_{0}\right) x_{j}=0, \\
x_{1} \neq 0, x_{j} \neq 0,(j>2) .
\end{array}\right.
$$

From here $x_{1}=\left(S_{0}+N\right) / 2$ and $x_{j}=\left(S_{0}-N\right) / 2$. Such points form a subset of the set of equilibrium points of the $n$-dimensional open Eigen's hypercycle model. Every point of this subset appears in nonnegative region of phase space, when $S_{0} / N \geq 1$. So, $S_{0} / N=1$ is a bifurcation value. The fact, that at least $n-2$ equilibrium points take part in the abovementioned bifurcation.

\section{ECOLOGICAL INTERPRETATION}

Bifurcations, connected with change of stability of equilibrium points, are especially interesting from practical point of view. Only two bifurcation correspond to this criteria: $\frac{S_{0}}{N}=\frac{1}{1+a_{1}}, \frac{S_{0}}{N}=\frac{1+a_{1}+a_{2}}{1+a_{2}+a_{1} a_{2}}$.

From the analysis of equilibrium points it was clear that there are four possible stable equilibrium points; during the bifurcations they can change each other, and in any case only one point are an attractor:

1. When $0<\frac{S_{0}}{N}<\frac{1}{1+a_{1}}$, point $P_{5}$ is stable. In this case the size of ecological niche is so small, that only one association is able to exist in the ecosystem.

2. When $\frac{1}{1+a_{1}}<\frac{S_{0}}{N}<\frac{1+a_{1}+a_{2}}{1+a_{2}+a_{1} a_{2}}$, point $P_{8}$ is stable.

With such values of the parameters (capacity of the environment) there are sufficient resources for the second association, which is in competition with the first one. 
3. When $\frac{1+a_{1}+a_{2}}{1+a_{2}+a_{1} a_{2}}<\frac{S_{0}}{N}<1+a_{1}+a_{1} a_{2}$, point $P_{11}$ is stable. In this case size of ecological niche is so big, that three associations are able to coexist in the biogeocoenose. And that they use all the resources.

4. When $1+a_{1}+a_{1} a_{2}<S_{0} / N<+\infty$, point $P_{4}$ is stable. This case corresponds to an excess of resources. In the ecosystem there are three associations, which reach their maximal possible sizes.

One of natural questions: why $S_{0} / N=1+a_{1}+a_{1} a_{2}$ is not bifurcation value? When capacity of the environment $S_{0}$ becomes larger than $\left(1+a_{1}+a_{1} a_{2}\right) N$, the topology of the system is not changed, although, formally, the new point becomes to be stable. Such situations are quite usual in mathematical investigations of equilibrium points and bifurcation.

Concerning the bifurcation value $S_{0} / N=1$, it is difficult to determine its practical role. One can assume that this critical rate delineates various ways to stabilise the system if it is out of its steady state.

\section{CONCLUSION}

In the contribution, the three-dimension open Eigen's hypercycle model (1) was considered. In the case of small (nonzero) size of the ecological niche, only one association is able to exist in the system. When capacity of the environment grows, the second and the third association are included in the ecosystem gradually. It is shown, that the process of origin of next associations is accompanied with bifurcations of system (1). When size of niche is $S_{0}>\left(1+a_{1}+a_{1} a_{2}\right) N$, there is excess of resources in the biogeocoenose. The situation of appearance of excess of resources is not bifurcation, because topology of the ecosystem does not change and development of the system is continuous.

As it clear from the research, the main parameter, which determines the behaviour of the system is the capacity of the environment $S_{0}$. Parameters $a_{1}$ and $a_{2}$ influence only on relationships between the associations.

Analysis of three-dimension open Eigen's hypercycle model, together with the two-dimension one (Chernyshenko, 1995), allows proposing of the following assumptions for $n$-dimensional case:

1 . In the process of ecosystem successions has $n$ stages. Each of them is characterised by activation of a new association. Number of "activated" associations is determined by size of the environment capacity $S_{0}$. If the size of niche is small $\left(0<S_{0}<N /\left(1+a_{1}\right)\right)$, only one association is able to exist in the ecosystem.

2. Each "activation" of a new association is accompanied with bifurcations of the system. It is interesting, that the first $n-2$ conditions of the activation are the same as for $(n-1)$-dimensional system.
3. There is excess of resources in the ecosystem when size of ecological niche is $S_{0}>N \sum_{i=0}^{n} \prod_{j=0}^{n-i} a_{j}, a_{0}=1$. Size of associations in this case is calculated as $x_{j}=N \prod_{i=0}^{j-1} a_{i}, a_{0}=1, j=\overline{1, n}$.

The proof of these assumptions is a matter of future research.

\section{REFERENCES}

Allen, P.M. 1976. "Evolution, population dynamics and stability". Proceedings of the National Academy of Sciences of the USA, Vol. 73, No. 3, 665-668.

Berezich, I.C. and N.P. Zitkov. 1959. Computing technique. Physico-mathematical literature main Press, Moscow.

Chakrabarti, C.G.; Ghosh Sutapa; and Bhadra Syamali. 1995. "Non-equilibrium thermodynamics of Lotka-Volterra ecosystems: Stability and evolution." Journal of Biological Physics, Vol. 21, Is. 4, 273-284.

Chernyshenko, S.V. 1995. "Qualitative analysis of twodimensional modification of the M. Eigen's hypercycle and internal catastrophes" Problems of applied mathematics and mathematical modelling. Dniepropetrovsk University Press, 129-134.

Chernyshenko, S.V. 2005. Nonlinear analysis of forest ecosystems dynamics. Dnipropetrovsk University Press, Dnipropetrovsk.

Culver, D.C. 1981. “On Using Horn's Markov Succession Model." The American Naturalist, Vol. 117, No. 4 (Apr), 572-574.

Eigen M. and P. Schuster. 1979. The Hypercycle. A principle of natural self-organization. Springer-Verlag, Berlin, Heidelberg, New York.

Kogan, A.B.; N.P. Naumov; B.G. Rezabek; and O.G. Chorajan. 1977. Biological cybernetics. Vysshaja shkola, Moscow.

Lepš Jan and Karel Prach. 1981. "A Simple Mathematical Model of the Secondary Succession of Shrubs." Folia Geobotanica \& Phytotaxonomica, Vol. 16, No. 1, 61-72.

Lippe E.; J.T. De Smidt; and D.C. Glenn-Lewin. 1975. "Markov Models and Succession: A Test from a Heathland in the Netherlands." Journal of Ecology, Vol. 73, No. 3 (Nov), 775-791.

Logofet, D.O. 1997. "Inhomogeneous Markov models for succession of plant communities: New perspectives on an old paradigm." Izvestiya Akademii Nauk. Seriya Biologicheskaya, 613-622.

Lourival R.; Martin Drechsler; Matthew E. Watts; Edward T. Game; and Hugh P. Possingham. 2011. "Planning for reserve adequacy in dynamic landscapes; maximizing future representation of vegetation communities under flood disturbance in the Pantanal wetland." Diversity and Distributions, Vol. 17, Is. 2 (Mar), 297-310.

Rabotnov, A.T. 1992. Phytocenology. MGU Press, Moscow.

Sukachev, V.N. 1972. Selected transactions: in three volumes. Vol. 1. Bases of forest typology and biogeocenology. Nauka, Leningrad.

Tilman D. 1990. "Constraints and Tradeoffs: Toward a Predictive Theory of Competition and Succession." Oikos, Vol. 58, No. 1 (May), 3-15.

Weis, J.J.; B.J. Cardinale; K.J. Forshay; and A.R. Ives. 2007. "Effects of species diversity on community biomass production change over the course of succession." Ecology, Vol. 88, No 4 (Apr), 929-939. 


\section{AUTHOR BIOGRAPHY}

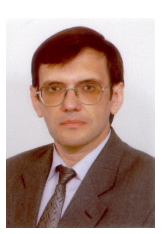

SERGE V. CHERNYSHENKO was born in Dnipropetrovsk, Ukraine and graduated from the Dnipropetrovsk State University. After obtaining $\mathrm{PhD}$ degree on computing in 1986, was, successively, head of Laboratory of Mathematical Modeling in Biology, head of Computer Science Department, dean of Applied Mathematics Faculty at Dnipropetrovsk National University; head of Department of Applied Mathematics and Social Informatics in Khmelnitsky National University. He was a supervisor of several Ukrainian national projects on mathematical modelling. From 2009 he is a visiting professor of Koblenz-Landau University, Faculty of Informatics. His e-mail address is svc@a-teleport.com and his web-site can be found at www.uni-koblenz.de/ svc

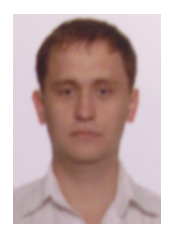

ROMAN V. RUZICH was born in Khmelnitsky, Ukraine and graduated from the Khmelnitsky National University. From 2010 he is postgraduate student of Khmelnitsky National University, Department of Applied Mathematics and Social Informatics. His e-mail address is ninasus@gmail.com. 